\title{
Evaluation of iMentors' Training Program of the Umbrella Project
}

\author{
Zachopoulou E. ${ }^{1}$, Vitoulis M. ${ }^{1}$, Grammatikopoulos V. ${ }^{1}$, Kotsakis R. ${ }^{1}$ \\ Tsigilis N. ${ }^{2}$ \& Gregoriadis A. ${ }^{2}$ \\ ${ }^{I}$ International Hellenic University, Greece \\ ${ }^{2}$ Aristotle University of Thessaloniki, Greece \\ Corresponding author’s email: ezachopo@ihu.gr
}

\begin{abstract}
The International Hellenic University (IHU) participates in a project partially funded by EU, named "Umbrella", aiming at providing the necessary psychological support and counseling, especially to disadvantaged students, towards the completion of their studies [1]. To this end, a network of academic experts were asked to select and train the iMentors, who are IHU students willing to support and help their counterparts. The purpose of the present study was to evaluate the iMentors' training program of the Umbrella project, in which 75 students participated. Their mean age was 22.2 years old $(\mathrm{SD}=3.89)$, and $66.7 \%$ were women $(\mathrm{N}=50)$. The iMentors are enrolled at 15 different departments of the IHU Alexandria Campus in Thessaloniki, Greece. An evaluation instrument comprised of ten items was developed specific for the purpose of the current study and was administered to participating students. Responses were given on a five-point Likert scale. Exploratory factor analysis indicated the retention of two reliable factors. Items designed to assess the "trainers" were loaded on the 1rst factor, whereas items designed to assess the "organization of the training" were loaded on the second factor (alpha values .876 and .896 respectively). The association between the two factors was positive, significant, yielding a strong value of .730. Descriptive statistics as well as one sample t-test showed that participants were very satisfied from the training program. Comparison of the two aspects of the training indicated that the iMentors were significantly more satisfied from the "trainers" factor than from the factor "organization of the training". The current findings seem to suggest the positive evaluation of students participating in the iMentors training program and gives us important information for the improvement of the "organization of the training" for future use.
\end{abstract}

Keywords: assessment; exploratory factor analysis; higher education; mentoring;

[1] Kotsakis R., Tsigilis N., Grammatikopoulos V., Zachopoulou E. and the research academic team of the project "Umbrella", "Supporting "vulnerable" students to complete their studies during the economic crisis in Greece: The Umbrella program of International Hellenic University", in Proceedings of the 23th International Conference on Interdisciplinary Social Sciences (ICISS 2021), Paris, France, Jan. 25-26, 2021 